\title{
CMOS Rad-Hard Front-End Electronics for Precise Sensors Measurements
}

\author{
Samuel Sordo-Ibáñez, Blanca Piñero-García, Manuel Muñoz-Díaz, Antonio Ragel-Morales, Joaquín \\ Ceballos-Cáceres, Luis Carranza-González, Servando Espejo-Meana, Alberto Arias-Drake, Juan \\ Ramos-Martos, José Miguel Mora-Gutiérrez, and Miguel Angel Lagos-Florido
}

\begin{abstract}
This paper reports a single-chip solution for the implementation of radiation-tolerant CMOS front-end electronics (FEE) for applications requiring the acquisition of base-band sensor signals. The FEE has been designed in a 0.35 $\mu \mathrm{m}$ CMOS process, and implements a set of parallel conversion channels with high levels of configurability to adapt the resolution, conversion rate, as well as the dynamic input range for the required application. Each conversion channel has been designed with a fully-differential implementation of a configurable-gain instrumentation amplifier, followed by an also configurable dual-slope ADC (DS ADC) up to 16 bits. The ASIC also incorporates precise thermal monitoring, sensor conditioning and error detection functionalities to ensure proper operation in extreme environments. Experimental results confirm that the proposed topologies, in conjunction with the applied radiation-hardening techniques, are reliable enough to be used without loss in the performance in environments with an extended temperature range (between -25 and $125^{\circ} \mathrm{C}$ ) and a total dose beyond 300 krad.
\end{abstract}

Index Terms-nuclear instrumentation and measurement; space applications; CMOS mixed-signal ASICs; front-end electronics; RHBD; dual-slope ADC.

\section{INTRODUCTION}

$\mathrm{T}$ HE front-end electronics (FEE) is a very common element in space, high energy physics and nuclear applications. This circuitry performs functions for sensor-signal conditioning, analogue-to-digital conversion (ADC), and interfacing with the higher levels of the system, but also has to be capable of overcoming the extreme environmental conditions, in terms of radiation and extended operating temperature range, while maintaining the performance. This implies the use of robust solutions and additional functionalities, like temperature monitoring, error detection or on-flight calibration functions [1].

Integrated circuits are widely employed in these applications to achieve improvements in the performance and, at the same time, reduce the weight, volume and power consumption. This is especially critical in space instrumentation, due to the current trends of costs reduction

The authors are with the Instituto de Microelectrónica de Sevilla, IMSECNM (CSIC/Universidad de Sevilla), Sevilla, Spain (e-mail: espejo@imsecnm.csic.es)

Color versions of one or more of the figures in this paper are available online at http://ieeexplore.iee.org.

Digital Object Identifier $x \times x \times x x x x x x x x x x$ and the proliferation of small-sized satellites.

The availability of recent radiation-tolerant field programmable gate arrays (FPGAs) allows the implementation of high performance digital systems within a single chip [2]. There are no equally efficient commercial off-the-shelf (COTS) solutions for analog or mixed-signal subsystems, like the FEE, so the use of application-specific integrated circuits (ASICs) becomes mandatory. However, most approaches reported in the literature are focused to fulfill very specific requirements or applications. In this scope, the design of mixed-signal ASICs with the objective to be applied in many similar applications, also referred to as application-specific standard products (ASSPs), is actually a research area of special interest and relevance in space instrumentation [3].

The traditional way of getting radiation-hardened integrated FEEs has been achieved by using specific technological processes [4]-[6]. However, this solution may become too expensive and sometimes with a performance delay with respect to the modern standard CMOS technologies. In addition, this solution is limited by a reduced number of foundries and also highly regulated by the export restrictions. An alternative solution, increasingly popular during the last decade, is the use of conventional CMOS processes with the application of radiation-hardening by design (RHBD) techniques, which are applied during the design flow [7].

One of the most common RHBD techniques is the implementation of specific transistor layout geometries, in combination with the use of guard rings, in order to minimize the leakage current that is induced by the accumulation of trapped holes in the lateral isolation oxide [8]. However, the ionizing radiation also induces the formation of trapped holes in the gate oxide, together with the accumulation of interface traps in the region between the gate oxide and the silicon [9]. These traps induce shifts in the threshold voltage of the MOS transistors, and also reduce the channel mobility, which yields in a degradation of both the transconductance and the noise performance [10]. In general, these effects are not mitigated with the use of specific transistor layout geometries [11], and additional solutions, e.g. at the architectural level, must be applied to address these issues.

At the circuit level, the progressive degradation of these transistor parameters produces variations in the $\mathrm{DC}$ and $\mathrm{AC}$ characteristics of the analogue circuits. The digital circuitry is also affected with an increase of the propagation delay, which 
may affect to the operating frequency and also to the correct functionality of the analogue section [12].

Several ADC architectures have been proposed in the literature for the implementation of CMOS FEEs in nuclear and space applications, including time-to-digital converters (TDCs) [13], sigma-delta ADCs ( $\Sigma \Delta$ ADCs) [14] and singleslope ADCs (SS ADCs) [15]. One of the most common observed effects at the circuit level is a significant degradation of the ADC gain error with the TID [14], [16], which directly affects the stability of the output code. This variation is associated to several effects. For the case of a conventional SS $\mathrm{ADC}$, the performance of the current source, which is commonly employed to generate the reference ramp signal, is affected by the induced variations in the transconductance and the threshold voltage, yielding in a progressive degradation of the ADC gain. In addition, the variation in the clock frequency, which is employed in the ramp generation, is also directly reflected in the gain of the ADC. When high levels of precision are aimed, this usually requires the use of calibration techniques [15], [17].

This paper reports the design of a mixed-signal ASIC that provides a reliable on-chip solution for the implementation of a standard FEE. The proposed design combines the use of RHBD techniques with robust topologies for signal acquisition to develop the multi-channel readout system in a standard 0.35 $\mu \mathrm{m}$ CMOS technology. In order to ensure high levels of stability of the output code with the TID, two ADC architectures are proposed. The first topology is based on a dual-slope conversion process, taking advantage of the inherent robustness of this type of conversion against the fluctuation of its components. The second topology is based on a SS ADC with the implementation of a feedback loop in the ramp generator that compensates the fluctuations induced by the TID, including the variations in the current source, as well as the variations in the clock frequency.

The FEE is highly configurable in order to be adapted to most common sensor applications, also including additional functionalities to ensure proper operation in extreme environments, like precise thermal monitoring, error detection and sensor conditioning functions.

The results of this work are being initially applied in the development of scientific instruments including, among others, magnetometers, accelerometers and temperature measurement systems for the Mars MetNet Precursor mission [18], and expected to be applied for the implementation of wind and solar irradiance measurement systems for the MEDA (Mars Environmental Dynamics Analyzer) instrument, which is a payload to be included in the rover of the Mars 2020 NASA mission [19].

Section 2 presents the system description of the proposed FEE. Section 3 provides a detailed circuit description of the sensor measurement system, disclosing the key design aspects. Additional functionalities, like temperature monitoring and sensor conditioning are described in Section 4. The RHBD techniques employed for the reliable operation in extreme environments are described in Section 5. The experimental performance and robustness of the designed FEE is presented

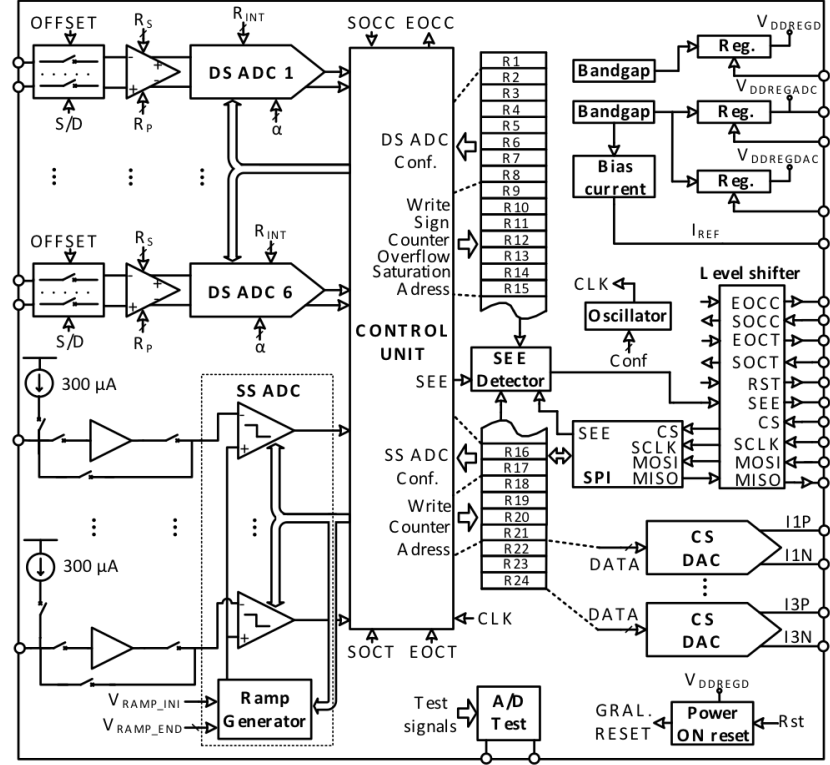

Fig. 1. Block diagram of the proposed FEE.

in Section 6. Finally, Section 7 presents the conclusions.

\section{ARChitecture Description of the Proposed FEE}

Fig. 1 shows the block diagram of the proposed rad-hard FEE. The ASIC includes six parallel dual-slope ADCs (DS ADCs) for accurate sensor measurements, a multichannel single-slope ADC (SS ADC) for temperature measurements, and three bipolar current-steering DACs (CS DACs) to perform sensor conditioning and calibration functions. A control unit is in charge of controlling the operation by implementing two finite state machines (FSMs), one for the DS ADCs and the other one for the SS ADC.

All the operation parameters and configuration options are programmed internally, with a total of 13 configuration registers of 16 bits. The digital output values are also stored in specific registers of the internal memory. The ASIC provides access to the memory for both loading configuration and reading output data by implementing an internal serial protocol interface (SPI). The clock frequency can also be programmed internally between 3 and $100 \mathrm{MHz}$ to control the operation of the whole FEE.

The ASIC can be supplied with a wide voltage range between 3.3 and $5.5 \mathrm{~V}$. The digital interface pins employ a dedicated voltage supply to support any logic family under that range. Three internal voltage regulators provide separated power supplies for ADCs, DACs and digital logic respectively, minimizing noise coupling. All voltage references in the chip are generated internally using the respective bandgap voltages to ensure temperature stability.

\section{Sensor Measurements with the DS ADCs}

Each of the six available signal-acquisition channels contains a preamplifier, and a DS ADC implemented with an integrator followed by a comparator. Fig. 2 shows the circuit schematic and the simplified timing diagram of the conversion cycle. 


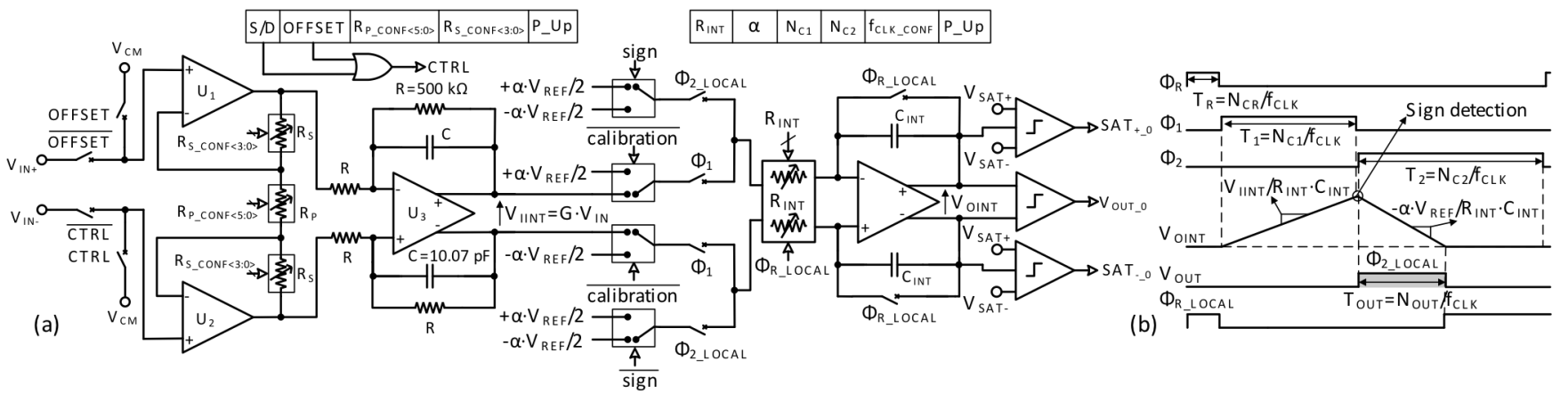

Fig. 2. (a) Schematic of the conversion channel for sensor measurements. (b) Timing diagram of one conversion cycle of the DS ADC.

Each conversion cycle is managed by the FSM through the signals $\Phi_{\mathrm{R}}, \Phi_{1}$ and $\Phi_{2}$, which are common to all the conversion channels. As explained in detail later, each conversion channel makes use of these signals to generate its own local control signals ( $\Phi_{R_{\_} L O C A L}$ and $\Phi_{2 \_ \text {LOCAL }}$ ) by using simple digital logic, and with the objective of keeping the integrator reset right after the commutation of the comparator ( $\mathrm{V}_{\text {OUT }}$ ) during $\Phi_{2}$. Thus, $\Phi_{2 \text { L LOCAL }}$ is activated with $\Phi_{2}$, but disabled when the comparator switches. This commutation can be a positive or negative edge, depending of the sign of the input signal. $\Phi_{R_{-} L O C A L}$ is activated when the ADC is in the reset phase $\left(\Phi_{R}=" 1 "\right)$ or waiting for a conversion cycle, and also when $\Phi_{2 \_ \text {LOCAL }}$ is disabled during $\Phi_{2}$.

The dual-slope architecture has been selected to provide high levels of noise rejection and accuracy. In addition, it is insensitive to the accurate value of its components, including the time constant of the integration phases as well as the accurate frequency of the internal clock. A first evidence of the inherent robustness of the dual-slope architecture against TID effects, and with respect to other ADC topologies, can be found in [20], where the authors report experimental results for commercial off-the-shelf (COTS) devices with a total dose up to $30 \mathrm{krad}$. The goal of this work is to achieve robustness beyond $300 \mathrm{krad}$.

The resolution of each conversion channel is configurable between 12 bits and 16 bits, with a corresponding conversion rate between $20 \mathrm{kS} / \mathrm{s}$ and $2.6 \mathrm{kS} / \mathrm{s}$. The input voltage range can be configured up to $\pm 2 \mathrm{~V}$. The ASIC also incorporates specific configuration options in order to work with both differential and single-ended input signals, as well as to perform offset measurements.

The first preamplification stage provides configurable gain, high common-mode and power supply rejection ratios (CMRR and PSRR), and input noise rejection. It also provides capacitive input impedance, which is necessary for many sensor signals. The preamplifier is implemented with a fullydifferential version of a 3-opamp instrumentation amplifier (IA) with low-pass filtering, and programmable gain (G), which can be set between 1 and 1000 by using two resistances:

$$
G=1+\frac{2 \cdot R_{S}}{R_{P}}
$$

where $R_{S}$ is given by $R_{S}=\left(R_{S_{-} \text {CONF }}+1\right) \times 15 \cdot 10^{3} \Omega$, and $R_{P}$ is equal to $\mathrm{R}_{\mathrm{P}}=3 \cdot 10^{4} / \mathrm{R}_{\mathrm{P}_{-} \text {CONF }} \Omega$. When $\mathrm{R}_{\mathrm{P}_{-} \text {CONF }}$ is equal to zero, the IA is configured as a source follower.
The integrator has also been implemented with a fullydifferential topology. The sign of the input signal is determined at the end of the first integration stage, which allows doubling the maximum input voltage range as well as the conversion rate of the acquisition channel. The integrator time constant is configurable by means of $\mathrm{R}_{\mathrm{INT}}$ (between 515 $\mathrm{k} \Omega$ and $4.096 \mathrm{M} \Omega$ ) in order to fully exploit the output signal swing in all the possible resolution configurations and, at the same time, avoiding saturation. The capacitor $\mathrm{C}_{\mathrm{INT}}$ is set to 40 $\mathrm{pF}$. The opamp uses a 2-stage Miller topology, as shown in Fig. 3.

The high speed of the comparator, up to $100 \mathrm{MHz}$, is achieved using a fully-differential regenerative latched approach, as shown in Fig. 4. A preamplifier stage in the comparator amplifies its input voltage difference, mitigating the offset voltage of the latch and reducing the kickback effect. The final stage is a regenerative latch that operates with the clock signal.

The final relationship between the input voltage and the output code can be expressed by [21]:

$$
N_{\text {OUT }}=\operatorname{sign}\left(V_{I N}\right) \cdot\left|\frac{G \cdot V_{I N} \cdot N_{C 1}}{\alpha \cdot V_{R E F}}\right|
$$

where $\mathrm{N}_{\mathrm{Cl}}$ is the duration, in clock cycles, of the first integration phase, and $\alpha \cdot V_{\mathrm{REF}}$ is the internal programmed

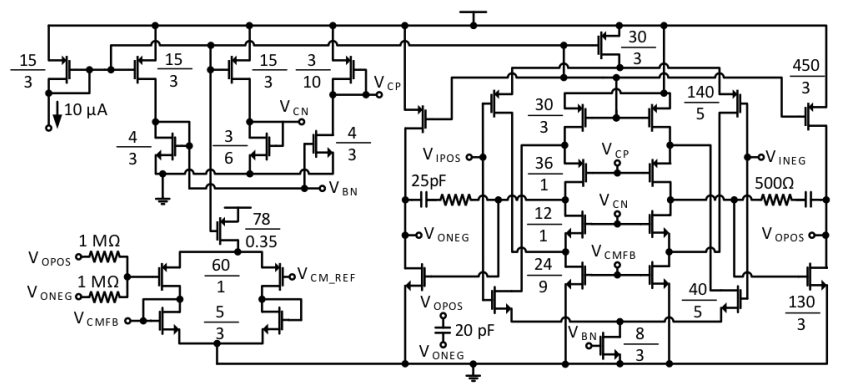

Fig. 3. Operational amplifier of the integrator.

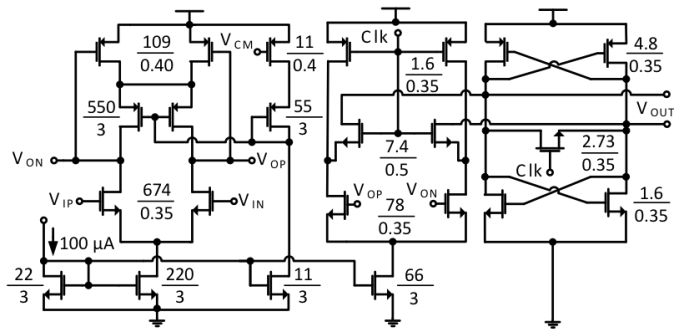

Fig. 4. Fully differential regenerative latch comparator with preamplifier. 
reference voltage for the de-integration phase. It is obtained from the general reference $( \pm 2 \mathrm{~V})$, which is scaled by the configurable parameter $\alpha$, yielding in effective reference values from $\pm 0.25 \mathrm{~V}$ up to $\pm 2 \mathrm{~V}$ (with steps of $0.250 \mathrm{~V}$ ). The conversion-channel resolution can be programmed by setting $\mathrm{N}_{\mathrm{C} 2}$, corresponding with the maximum duration, in clock cycles, of the de-integration phase $\left(\mathrm{N}_{\mathrm{C} 2}=2^{(\mathrm{RES}-1)}-1\right)$.

As can be seen in (2), the relationship between $V_{I N}$ and $\mathrm{N}_{\text {OUT }}$ is independent of both the frequency of the oscillator and the integration time constant, achieving high insensitivity to their variations, including the TID effects. Thus, the proposed design takes the advantage that all the induced variations are expected to be the same during the integration and the deintegration phases, assuming that the integration time constant is lower enough with respect to the time constant of the TID degradation, and their effects are then cancel out. The variation of the stability of the output code with the TID is only limited by the fluctuations of $\mathrm{V}_{\mathrm{REF}}$, which in turns depends on the variation of the bandgap voltage with the TID. This is an advantage, in terms of TID robustness, with respect to the conventional single-slope ADCs.

The equivalent voltage of the least significant bit (LSB) is given by:

$$
L S B=\frac{\left|\alpha \cdot V_{R E F}\right|}{G \cdot N_{C 1}}
$$

The capacity to program the gain $\mathrm{G}$, as well as $\mathrm{f}_{\mathrm{CLK}}, \mathrm{R}_{\mathrm{INT}}, \alpha$, $\mathrm{N}_{\mathrm{C} 1}$ and $\mathrm{N}_{\mathrm{C} 2}$, allows the conversion channel to adapt the resolution, conversion rate, as well as the dynamic input range for the required application. An example of configuration that maximizes the input voltage range is given by:

$$
\begin{gathered}
N_{C 1}=\left|\frac{V_{S A T_{-} I N T}}{V_{S_{S A} P R E}} \cdot f_{C L K} \cdot R_{I N T} \cdot C_{I N T}\right| \cong\left|f_{C L K} \cdot R_{I N T} \cdot C_{I N T}\right| \\
\alpha=\left|\frac{V_{S A T \_I N T} \cdot f_{C L K} \cdot R_{I N T} \cdot C_{I N T}}{\left(2^{R E S-1}-1\right) \cdot V_{R E F}}\right| \cong \frac{N_{C 1}}{N_{C 2}}
\end{gathered}
$$

where $\mathrm{V}_{\text {SAT_PRE }}$ and $\mathrm{V}_{\text {SAT_INT }}$ are the saturation levels of the IA and the integrator, respectively. In this design both values are equal to $\pm 2 \mathrm{~V}$. As can be seen in Fig. 2, two level comparators are in charge to monitor and alert from a saturation condition in the integrator. The FSM also alerts in a specific output register when an overflow condition is produced in the counter during the de-integration phase. These functionalities not only alert from any possible error, but also allow the implementation of auto-ranging techniques when used together with the programmable gain of the IA.

Fig. 5 shows the schematic of the relaxation oscillator to generate the clock signal with a programmable frequency with values between 3 and $100 \mathrm{MHz}$.

\section{A. Parallel data acquisition}

In order to reduce the area needed for the implementation, only one FSM is in charge of controlling the six conversion channels. It only needs three signals to manage the six conversions: $\Phi_{\mathrm{R}}$ for the reset phase, and $\Phi_{1}$ and $\Phi_{2}$ for the first and second integration phases, respectively. An additional

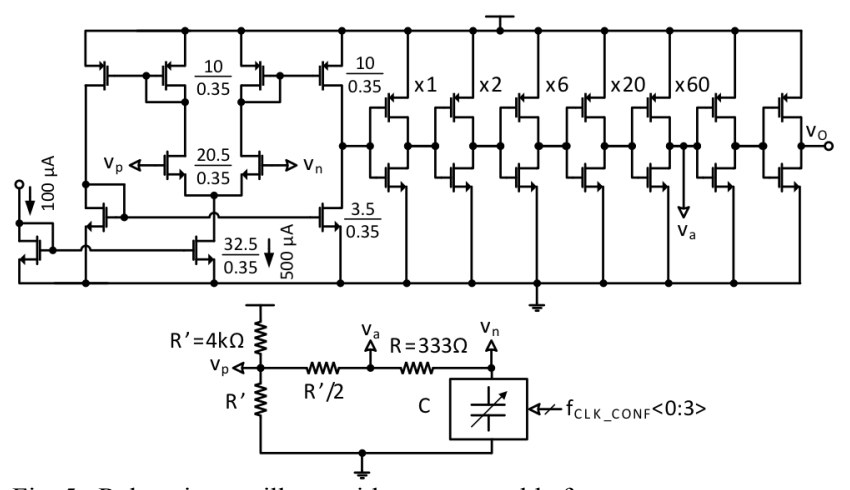

Fig. 5. Relaxation oscillator with programmable frequency.

signal (Init) is used to indicate to the analog part when the FSM is waiting for a start-of-conversion pulse (SOC). The effective time duration of the second integration phase, until the commutation of the comparator $\left(\Phi_{2 \_ \text {LOCAL }}\right)$, is dependent of the magnitude of the input signal, and then it can be different for each of the conversion channels. The maximum time duration of $\Phi_{2 \_ \text {LOCAL }}$ is then the duration of $\Phi_{2}$.

In order to avoid the reference voltage to be integrated during the period of time between $\Phi_{2 \_ \text {LOCAL }}$ and $\Phi_{2}$, a simple digital circuitry, shown in Fig. 6, is used locally in each of the conversion channels to generate the $\Phi_{2 \_ \text {LOCAL }}$ signal, and then, the $\Phi_{R_{-} L O C A L}$ signal that ensures the integrator is reset after the commutation of the comparator during $\Phi_{2}$, avoiding any possible saturation condition, and also reducing the effects of the parasitic capacitance of $\mathrm{R}_{\mathrm{INT}}$, as shown in detailed later. The integrator is also reset when the Init signal is activated by the FSM. A detailed time diagram of the conversion process, for the case of two conversion channels, is shown in Fig. 7.

\section{B. Noise rejection}

Several sources of noise must be considered in the operation of the integrator, including the sampling noise in the capacitor, as consequence of the reset phase, the noise generated by the resistor and the opamp, as well as the noise of the input signal and the reference voltage. The noise of the comparator as well as the jitter of the oscillator must also to be taken into account.

The most significant source of noise was the contribution of the resistance $\mathrm{R}_{\mathrm{INT}}$. Both the thermal noise from $\mathrm{R}_{\mathrm{INT}}$ and the noise from the sensor-signal (previously filtered by the preamplifier) are filtered by the integrator. The $\mathrm{RC}$ integration of an input voltage during a fixed amount of time is equivalent to a filter with a sinc function in the frequency domain, as shown in Fig. 8. Using the concept of equivalent noise bandwidth, the integrated noise power spectral density at the output of the integrator can be approximated by:

$$
S_{n O} \approx \frac{4 \cdot K \cdot T \cdot R_{n} \cdot T_{I N T}}{R_{I N T} \cdot C_{I N T}}
$$

where $\mathrm{K}$ is the Boltzmann constant, $\mathrm{T}$ is the temperature, $\mathrm{T}_{\mathrm{INT}}$ is the effective integration time, and $R_{n}$ is the equivalent noise resistance at the input of the integrator. For the case of the noise of $R_{I N T}\left(R_{n}=R_{I N T}\right), T_{I N T}$ is equal to the total time duration of $\Phi_{1}$ and $\Phi_{2}$ LOCAL. For the case of the noise of the input signal, $\mathrm{T}_{\mathrm{INT}}$ is equal to the time duration of $\Phi_{1}$.

With respect to the noise of the opamp, the transfer function 
of the integrator with respect to the input of the opamp $\left(\mathrm{H}_{2}(\mathrm{~s})\right)$ is multiplied by a zero located in $1 /\left(2 \cdot \pi \cdot \mathrm{R}_{\mathrm{INT}} \cdot \mathrm{C}_{\mathrm{INT}}\right) \mathrm{Hz}$, which cancels the pole of the sinc function, being the bandwidth of the opamp the only limiting factor of the noise folding, as shown in Fig. 9. The transistors of the differential pair were

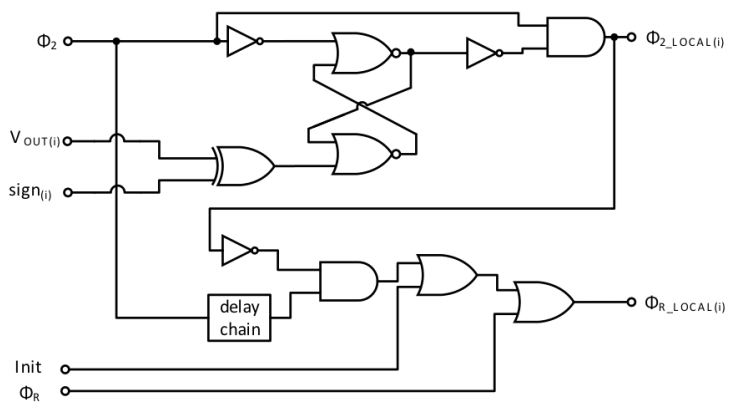

Fig. 6. Digital logic for local reset signal generation.

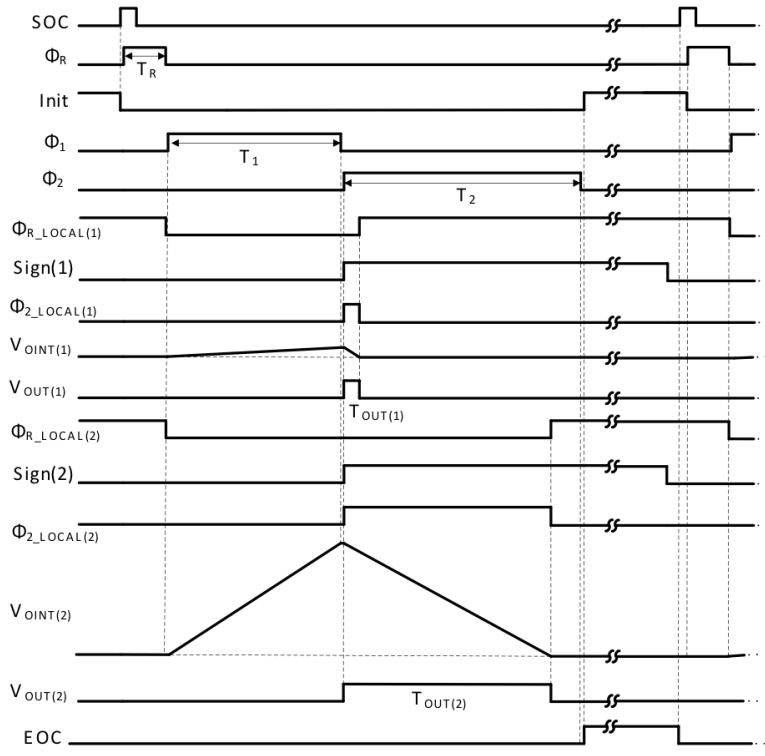

Fig. 7. Time diagram of parallel data conversion with 2 DS ADCs.

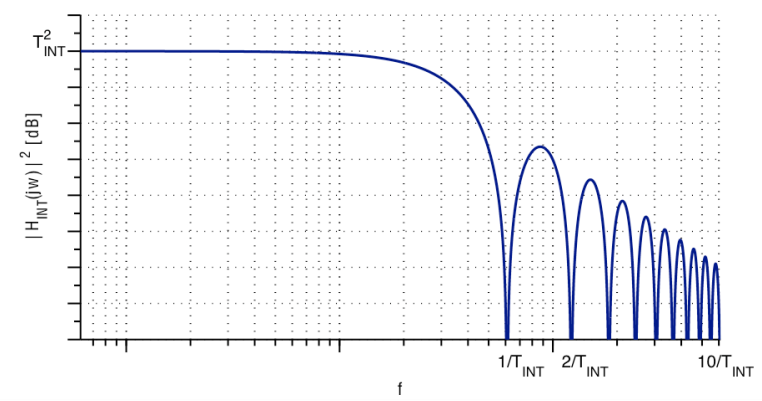

Fig. 8. Transfer function of the finite-time integration.

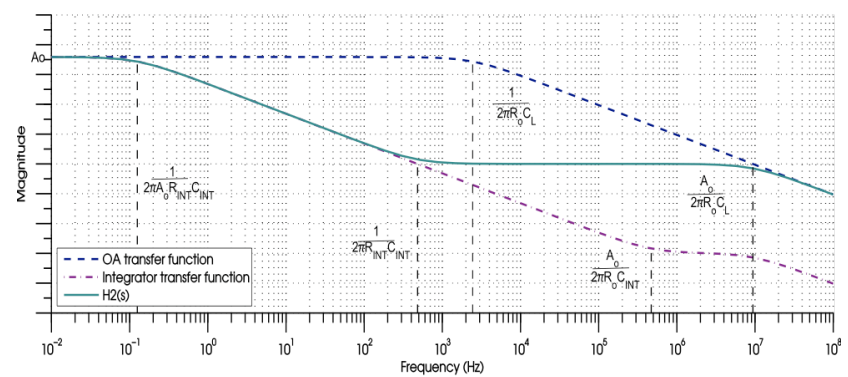

Fig. 9. Bode diagram of the transfer functions related with the integrator. the main contributors to the noise of the opamp, so they were designed with a high transconductance.

\section{Dynamic $N$-well biasing for linearity improvements}

The parasitic capacitances between the resistance $\mathrm{R}_{\text {INT }}$ and the substrate can introduce a significant non-linearity error, as well as a zero-cross distortion and a differential offset in the transfer characteristic of the DS ADC. These errors become more critical as the programmed value of $R_{I N T}$ is higher. In order to minimize these effects, the resistors of the integrator are placed in n-well regions, and an auxiliary resistance network, with lower values for the resistors, is used to properly polarize the n-wells, and to provide a lower time constant in order to keep the voltages of the parasitic capacitances constant.

Fig. 10 shows a simplified circuit schematic with the proposed auxiliary network for the dynamic N-well biasing. During the reset phase of the conversion cycle $\left(\Phi_{\mathrm{R}_{-} \text {LOCAL }}\right)$, all the switches in Fig. 10 are $\mathrm{ON}$ and the node $\mathrm{R}_{\mathrm{INT} \text { IN }}$ is

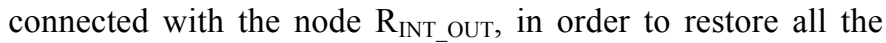
parasitic capacitances from any possible residual charge during previous conversions. Fig. 11 shows simulation results of the effect of the parasitic capacitance of $\mathrm{R}_{\mathrm{INT}}$ in the transfer characteristic of the DS ADC with and without the use of the dynamic N-well biasing. As can be seen, the use of this technique eliminates the effects of the parasitic capacitances of $\mathrm{R}_{\mathrm{INT}}$, significantly improving the linearity of the DS ADC.

\section{Improving the transient response of the integrator}

The step response of the integrator is relevant due to the transient switching between the integration phases required during every conversion process. The finite transconductance of the opamp as well as its finite bandwidth (BW) can introduce non-idealities in the transfer characteristic of the DS ADC. This is illustrated in Fig. 12, which shows simulation results of the behavior of the output voltage of the integrator

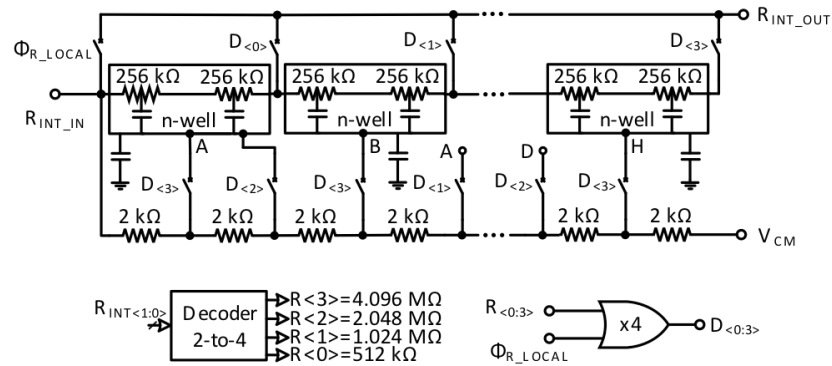

Fig. 10. Circuit schematic for the dynamic $\mathrm{N}$-well biasing in $\mathrm{R}_{\mathrm{INT}}$.

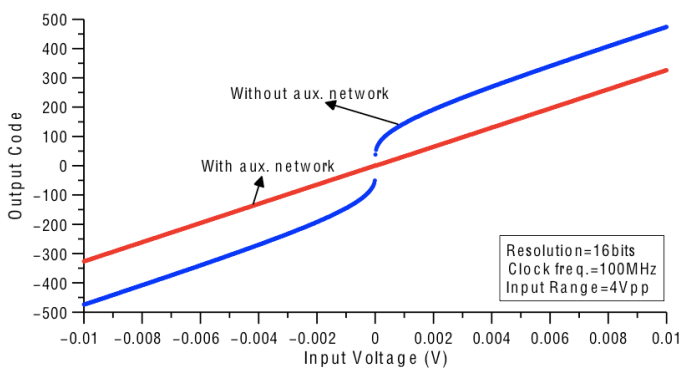

Fig. 11. Simulation of the effect of the parasitic capacitance of $R_{\mathrm{INT}}$ with and without the auxiliary resistance network $\left(\mathrm{R}_{\mathrm{INT}}=4.096 \mathrm{M} \Omega\right)$. 


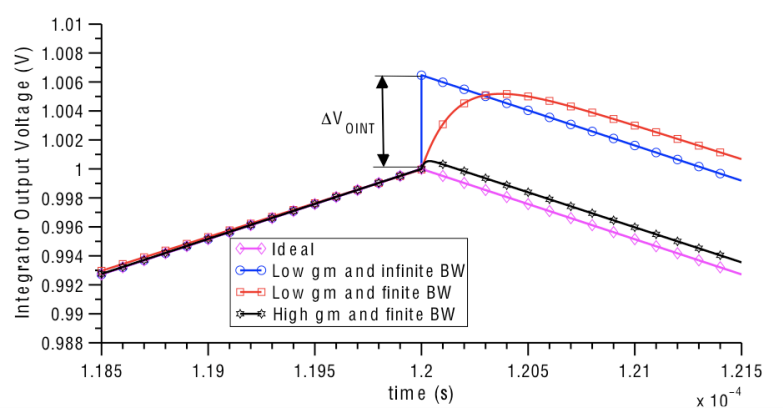

Fig. 12. Comparative simulation of the influence of the finite transconductance of the opamp in the transient response of the integrator.

when switching from the first integration stage to the second one, and for several models of the opamp. These non-idealities can yield in a differential offset, a zero cross distortion and non-linearity errors, since the transient response is dependent of the step amplitude, which in turn depends of the input signal. For the case of finite transconductance and infinite $\mathrm{BW}$, the value of $\Delta \mathrm{V}_{\text {OINT }}$ in Fig. 12, is given by:

$$
\Delta V_{\text {OINT }}=\frac{\Delta V_{I N T}}{g_{m} \cdot R_{I N T}}
$$

where $\Delta \mathrm{V}_{\text {INT }}$ is the amplitude of the input step and $g_{m}$ is the transconductance of the opamp. As discussed previously, the $\mathrm{BW}$ of the opamp cannot be too large, due to noise concerns, and then, it is needed to ensure a high transconductance in order to keep these non-idealities under the requirements. The total transconductance of the designed opamp is given by the gain of the first (folded-cascode) stage $(90 \mathrm{~dB})$ multiplied by the transconductance of the output stage $(1.9 \mathrm{mS})$, being high enough to ensure that the effect of the transient response is reduced to a minor systematic differential offset. This offset has no dependence with mismatch variations, being also independent of the magnitude of the input signal. The lowest value that was achieved during the design process was \pm 12 LSBs.

\section{TEMPERATURE MONITORING $\&$ SENSOR CONDITIONING}

One of the most common sources of error in sensor measurements is the drift of both the sensitivity and the offset with the temperature. This becomes more critical in environments with an extended temperature range. The general reference voltage of the ADCs $\left(\mathrm{V}_{\mathrm{REF}}\right)$ is externally accessible, and can be used to supply the external sensors in order to perform ratiometric measurements. This eliminates the influence of the bias voltage, including temperature drifts, in the relationship between the physical magnitude and the digital output code.

In order to perform more precise corrections, the ASIC also incorporates a SS ADC for temperature monitoring. Four PT1000 temperature sensors have been used in the first applications. This ADC is also highly configurable with resolutions between 10 and 15 bits, and with a corresponding conversion rate between 77 and $3 \mathrm{kS} / \mathrm{s}$. The input voltage range can also be configured with many ranges between 0 and $2.8 \mathrm{~V}$. High impedance paths can be connected at the input of

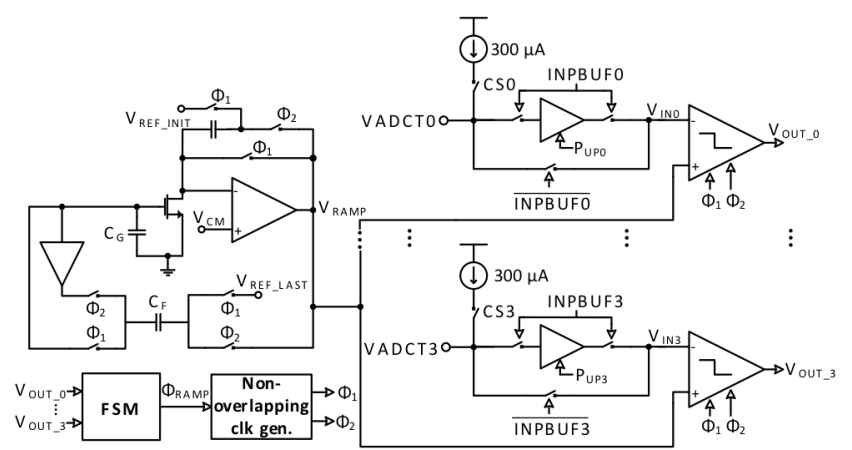

Fig. 13. Schematic of the SS ADC for temperature monitoring.

each channel when required. The implementation consists of parallel readout architecture with a ramp generator and four self-biased comparators [22], as shown in Fig. 13.

The generation of the ramp is implemented with an integrator driven by a voltage-controlled current source (NMOS transistor). As stated in the introduction, in a traditional SS ADC the gain and the stability of the output code depend on the induced fluctuations associated with the current source, as well as the variations in the clock frequency. In order to address these effects, the ramp generation is regulated by an automatic control of the gate voltage of the current source. This is done through the feedback loop implemented with the capacitor $\mathrm{C}_{\mathrm{F}}$ in Fig. 13, which adapts the final value of the ramp until it equals to the reference programmed value ( $\left.\mathrm{V}_{\text {REF_LAST }}\right)$. This loop adaption ensures that the ramp signal remains stable between its initial and final reference values, and independently of the induced variations by the TID. Thus, if the TID induces a reduction in the clock frequency, then the effective time-duration of the ramp generation will be increased, together with the final value of the generated ramp. This is automatically addressed with the implemented negative feedback loop by reducing the gate voltage of the current source, so the final value of the generated ramp is self-adjusted until it reaches again the reference value. This adaption ensures that the only source of variation in the ramp generation is the fluctuation of the reference voltages that define the ramp excursion, which are obtained from the bandgap circuit.

Most precise sensors employ a Wheatstone bridge configuration. The common method to perform measurements from a resistive Wheatstone bridge is the deflection mode, which consists of measure the differential output voltage of the bridge. However there are some types of sensors that exhibit a better performance using comparison methods, which mainly consist of a feedback loop configuration that provides a control signal to condition the bridge in order to keep it balanced. The control signal is then a direct measure of the physical quantity.

The proposed FEE allows the capability to perform measurements with both open loop (deflection mode) and feedback (comparison mode) methods. For feedback measurements, the FEE includes 3 monotonic binary-weighted CS DACs to balance the output voltage of the Wheatstone bridge. Fig. 14 shows an application example of the ASIC for the implementation of a magnetometer with anisotropic 
magnetoresistive sensors in a feedback configuration [23]. The conversion channel (preamplifier plus DS ADC), as observation element, closes the feedback loop. The CS DACs can also be used for additional functionalities, like offset correction or on-flight calibration functions.

As shown in Fig. 14, each CS DAC has a resolution of 8 bits plus sign. The full-scale output current is $\pm 25 \mathrm{~mA}$. Cascoded current sources have been used in order to increase the output impedance, minimizing the current variation due to the channel-length modulation. Due to area concerns, PMOS devices were used to implement the current sources, as NMOS transistors employ ringed-source layout (as shown in the next section), with higher area penalty. An external resistor with low temperature coefficient is used in combination with the internal bandgap in order to generate the reference current for the CS DACs.

\section{RHBD TECHNIQUES}

The proposed topologies for sensor data acquisition and temperature monitoring are oriented to increase the robustness in the sense to deal with the expected variations of some transistor parameters (e. g. the transconductance and the threshold voltage) with the TID effects and the temperature. However, it is needed to apply additional techniques in order to increase the robustness against other TID effects, like the induced leakage current, and also against the single-event effects (SEE).

Previous work to the design of this rad-hard ASIC was needed in order to characterize the selected standard $0.35 \mu \mathrm{m}$ CMOS process against radiation and the extended temperature range, including several irradiation campaigns with their corresponding test chips and experimental setups [24]. As part of this process, a basic radiation-hardened digital library was also designed.

The specific RHBD techniques applied in this ASIC included the use of NMOS transistors with ringed-source layout to improve the robustness against the induced leakage current. A layout example of the custom pcell is shown in Fig. 15. PMOS devices use the standard layout. Guard rings have been employed in every transistor (NMOS and PMOS) in order to minimize the probability of single-event latchup (SEL), also improving the robustness against TID, especially in NMOS transistors, as the $\mathrm{P}+$ guard ring isolates from adjacent NMOS transistors, supressing the induced intertransistor leakage current.

The chip has significant digital components, including 2 FSMs and the SPI, so additional techniques were applied in order to detect the single-event upsets (SEUs) and minimize their effects. Within this objective, both the FSMs as well as the SPI interface were implemented by using the one-hot encoding. In addition, all the memory elements are redundant having a specific error flag which alerts of a SEU event in a global output pin. In order to restore from any possible error during a conversion, the FSMs are always reset when a new start-of-conversion pulse is received.

Some additional amendments were required during the design flow, including the layout $\mathrm{p}$-cell for the ringed-source

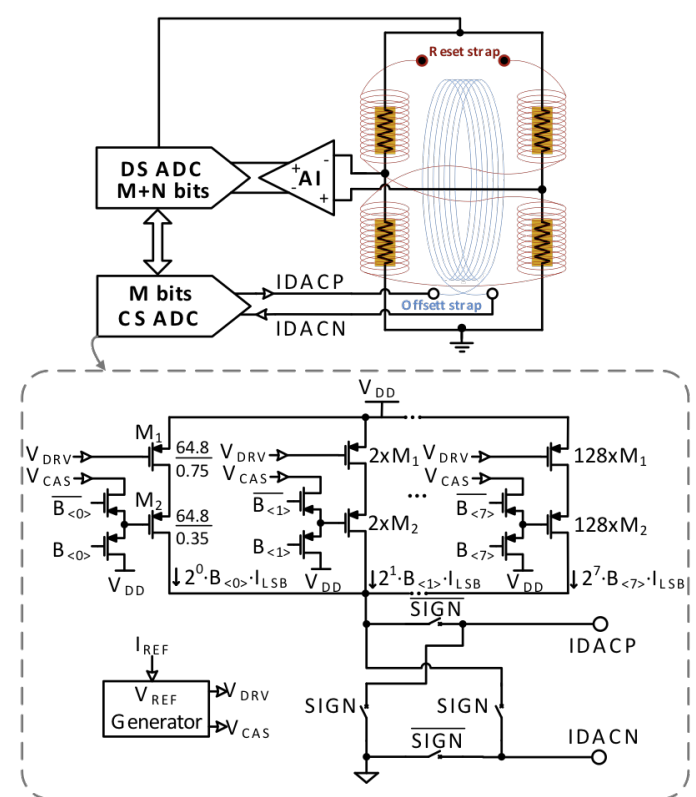

Fig. 14. Schematic of the CS DAC for feedback measurements (bottom) and example of application with a magnetoresistive sensor (top).

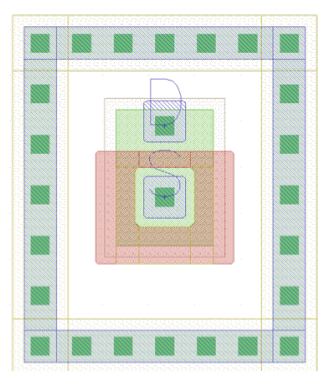

Fig. 15. Layout example of the p-cell designed for the ringed-source NMOS transistor with guard ring.

NMOS transistor, with the corresponding symbol and the electrical model to compute the equivalent transistor width. Additional layout rules and layers, as well as modifications in the scripts for the layout-versus-schematic (LVS) and the parasitic extraction were needed for the proper verification of the layout.

\section{EXPERIMENTAL RESULTS}

The proposed rad-hard FEE has been fabricated in the 0.35 $\mu \mathrm{m}$ standard CMOS process from Austria Microsystems (AMS). A microphotograph of the chip is shown in Fig. 16, with a total area of $4.5 \times 4.5 \mathrm{~mm}^{2}$. Each of the six readout channels for sensor mesurements has a total power consumption of $13 \mathrm{~mW}$, while the multichannel SS ADC has a total power consumption of $8 \mathrm{~mW}$.

Table I and Table II show, respectively, the experimental results of the performance of the sensor readout system (IA plus DS ADC) and the SS ADC for several resolution configurations. The results have been obtained at room temperature, with unity gain for the IA, maximum input voltage ranges, and with the maximum frequency of the oscillator, as most demanding configurations.

With respect to the DS ADC, the high transconductance of the opamp of the integrator, as well as the use of the dynamic 


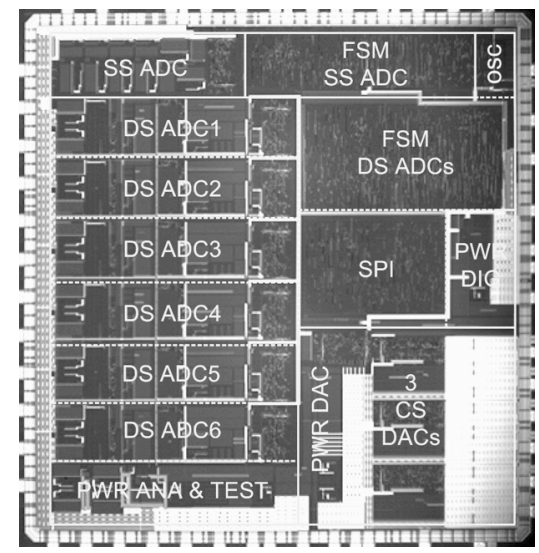

Fig. 16. Microphotograph of the fabricated chip.

TABLE I

EXPERIMENTAL RESULTS OF THE CONVERSION CHANNEL (IA + DSADC)

\begin{tabular}{cccccc}
\hline \hline Resolution $(\mathrm{bits})$ & 12 & 13 & 14 & 15 & 16 \\
\hline Conv. time $(\mu \mathrm{s})$ & 50 & 70 & 110 & 195 & 380 \\
$\mathrm{INL}$ & +0.1 & +0.2 & +0.6 & +0.7 & +0.9 \\
& -0.1 & -0.1 & -0.3 & -0.5 & -1.1 \\
DNL & +0.1 & +0.1 & +0.2 & +0.4 & +0.4 \\
& -0.1 & -0.2 & -0.2 & -0.3 & -0.4 \\
Root-mean-square noise & 0.1 & 0.1 & 0.5 & 0.5 & 0.9 \\
\hline \hline
\end{tabular}

Results in LSBs

TABLE II

EXPERIMENTAL RESULTS OF THE SS ADC

\begin{tabular}{ccccccc}
\hline \hline Resolution (bits) & 10 & 11 & 12 & 13 & 14 & 15 \\
\hline Conv. time $(\mu \mathrm{s})$ & 13 & 22 & 43 & 85 & 166 & 330 \\
INL & +0.4 & +0.2 & +0.3 & +0.9 & +3.9 & +14 \\
& -0.5 & -0.3 & -0.3 & -0.9 & -3.1 & -9.6 \\
DNL & +0.1 & +0.1 & +0.2 & +0.3 & +0.4 & +0.5 \\
& -0.1 & -0.1 & -0.2 & -0.2 & -0.3 & -0.4 \\
Root-mean-square noise & 0.01 & 0.5 & 0.5 & 0.5 & 0.9 & 1.8 \\
\hline \hline
\end{tabular}

Results in LSBs

well biasing technique, have reduced the effect of the nonidealities in the crossing-zero of the transfer characteristic of the DS ADC to a minor differential offset of \pm 12 LSBs for the maximum resolution. The DACs, implemented for the sensor conditioning, exhibit a monotonic transfer characteristic with an INL error below $0.3 \%$ of the full-scale.

In order to check the performance and robustness against TID effects, an irradiation campaign was performed using a standard gamma-ray source (Co-60 irradiation with a Gammabeam X200). The tests were carried out at the facilities of the Centro Nacional de Aceleradores (CNA-CSIC) in Seville (Spain). Four ASIC samples were tested up to a total dose of $320 \mathrm{krad}$ (733 hours at $220 \mathrm{rad} /$ hour followed by 133 hours at $1200 \mathrm{rad} /$ hour). The irradiation process was only disrupted once each week, during 6 hours, for taking measures. No annealing was applied after the irradiation as worst condition.

The ASIC exhibited very high levels of stability and robustness against TID effects. Fig. 17 and Fig. 18 show with detail the evolution against TID of the main metrics involved in the performance of the ASIC. As can be seen in Fig. 17 (a),

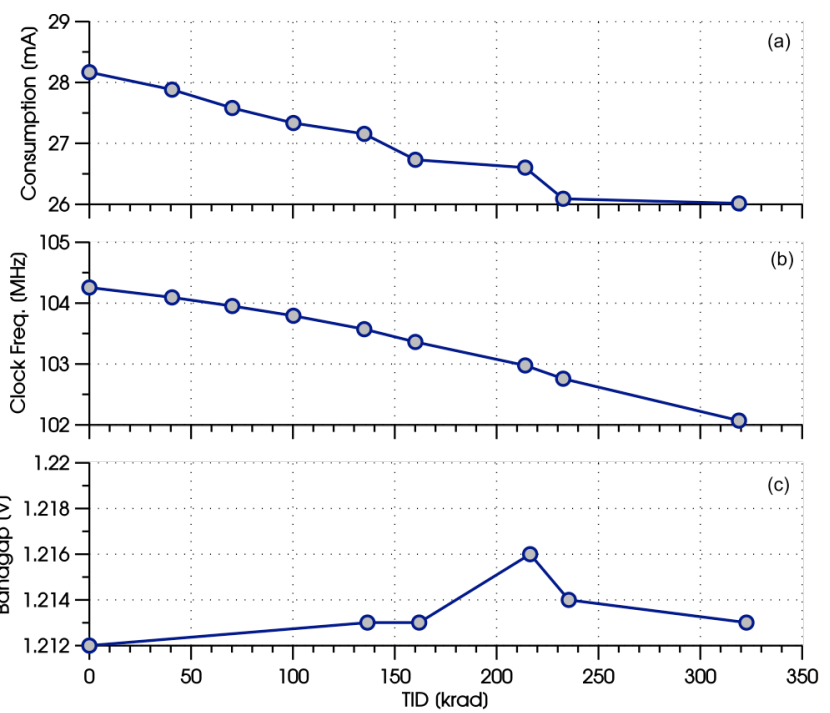

Fig. 17. Characterization results against TID.
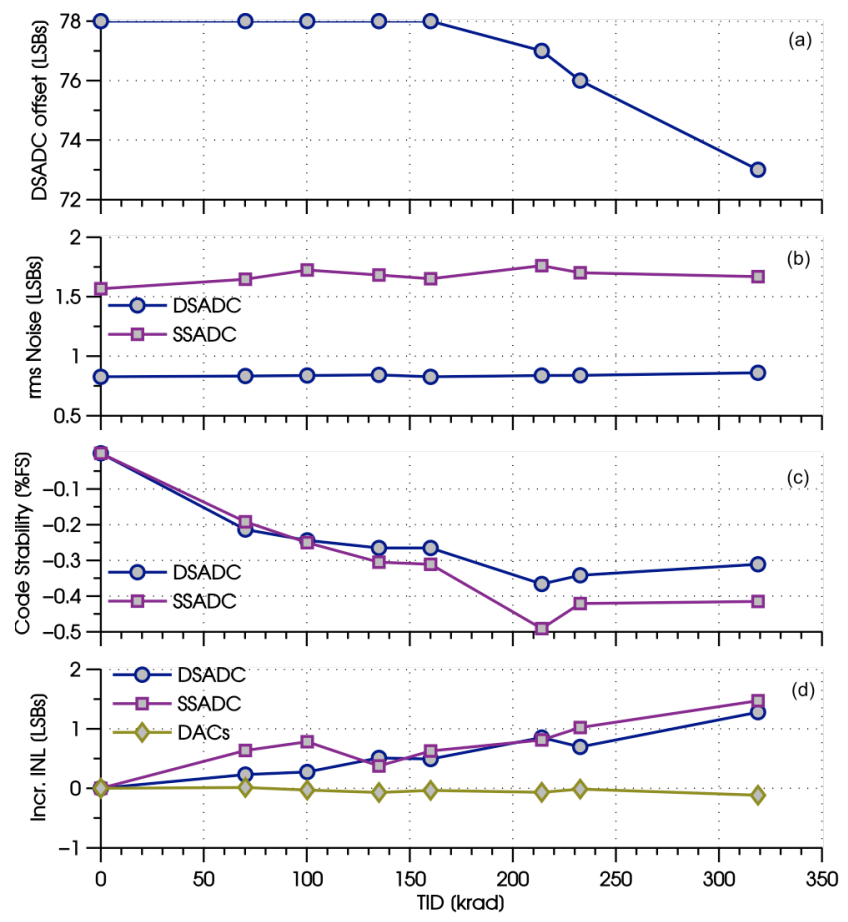

Fig. 18. Characterization results of the ASIC performance against TID.

the total power consumption of the ASIC decreases with the TID. This reduction, which is under the expected margins due to the previous characterization of the technology [24], is associated with an increment of the magnitude of the threshold voltage in the thick-oxide transistors (mainly PMOS transistors), together with a reduction in the transconductance of both thick- and thin-oxide (PMOS and NMOS) transistors. Due to power supply requirements, high-voltage (thick-oxide) transistors were employed for the bandgap reference generators, and also for the low dropout (LDO) regulators. The observed reduction in the current consumption also means that the ringed-source NMOS transistors, as well as the employed guard-rings, to prevent from the radiation-induced leakage current, are working properly.

Fig. 17 (b) and (c) show, respectively, the evolution with 
the TID of the internal clock frequency (programmed at 100 $\mathrm{MHz}$ ) and the evolution of the bandgap voltage, used for both the DS ADCs and SS ADCs. The observed reduction in the internal clock frequency, up to a $2.5 \%$, is associated with an increment in the propagation delay of the inverter chain that regenerates the output signal of the comparator in the relaxation oscillator (see Fig. 5), and mainly due to a reduction of the transconductance in these inverters.

The observed non-monotonic trend in the bandgap voltage is mainly associated with the variations of both the gain and the leakage current in the bipolar transistors. Similar behaviors have been reported by other works [25], [26]. There is also certain dependence with the dose rate [27], which tends to increase the step effect when switching to a higher dose rate. On the other hand, thick-oxide transistors are more sensitive to TID effects, especially when switching to a higher dose rate, and mainly due to the increase of hole-trapping in the gateoxide. This change in the dose rate can then produce abrupt variations in the threshold voltage of the MOS transistors, including a possible rebound effect in NMOS transistors. These variations can also contribute with non-monotonic fluctuations in the bandgap reference voltage.

In a conventional single-slope ADC, a variation of a $2.5 \%$ in the clock frequency would be directly reflected with the same magnitude in the stability of the ADC output code. As can be seen in Fig. 18 (c), even though the variations in the oscillator frequency, and the possible displacements in the DC operating points of the analogue circuits, associated with the reduction of the current consumption, the stability of the output code with the TID remains below than $0.5 \%$ for both topologies. This is due to the inherent insensitivity of the dualslope architecture to the fluctuation of its components. With respect to the SS ADC, the use of the proposed feedback loop also ensures the insensitivity against these fluctuations. The variation of the stability of the output code with the TID in Fig. 18 (c) correlates with the fluctuation of the reference voltage, and then it is only limited by the variations of the bandgap voltage. The use of ratiometric measurements allows canceling this influence, further improving the stability of the output code against TID.

The slight variation of the equivalent offset of the DS ADC with TID, shown in Fig. 18 (a), can be easily corrected by using the included offset measurement functionality. With respect to the noise performance, as shown in Fig. 18 (b), the root-mean-squared (rms) equivalent output noise of both ADC topologies does not suffer of significant deviation with respect to the non-irradiated case.

Fig. 18 (d) shows the increment of the integral-non-linearity (INL) error against TID with respect to the pre-irradiation case, and for the DS ADC, SS ADC and the CS DACs. The main contribution to the induced INL error in the ADCs was associated with a reduction in the output swing of the operational amplifiers, mainly due to a degradation of both the transconductance and the threshold voltage in the transistors of the output stages. For both ADC topologies, the incremental INL error is lower than 1.5 LSB for the maximum TID and without performing annealing. Fig. 19 shows with more

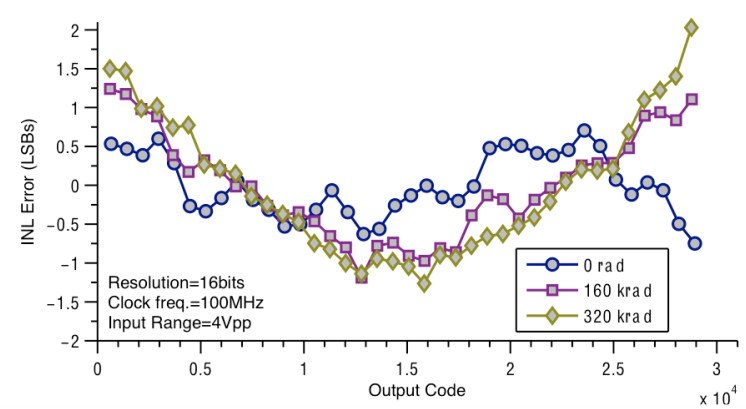

Fig. 19. Example of the evolution of the DS ADC linearity against TID.

detailed an example of the evolution of the INL error with TID for the case of the DS ADC. With respect to the CS DACs, with lower resolution, the incremental INL error is negligible.

Two samples of the ASIC were also characterized against SEEs with a heavy-ion source at the Cyclotron Resource Centre from the Université de Louvain-la-Neuve.

The irradiation consists of an ion cocktail. For the case of ions with a low linear-energy transfer (LET), the effective flux was 10 kions $\cdot \mathrm{cm}^{-2} \cdot \mathrm{s}^{-1}$, reaching a maximum fluence of $10^{7}$ ions $\mathrm{cm}^{-2}$. For the case of ions with a higher LET, the effective flux was 5 kions $\cdot \mathrm{cm}^{-2} \cdot \mathrm{s}^{-1}$, with a maximum fluence of $5 \cdot 10^{6}$ ions $\cdot \mathrm{cm}^{-2}$.

The characterization against SEUs was performed using the internal configuration registers (208 configuration bits) as test vehicles. During ion irradiation, the value of these registers was checked periodically with a rate of 35 times/s. Every discrepancy error, with respect to the original configuration, was considered a SEU, independently of its detection by the internal SEU detector. Multiple errors in the same reading were considered to be induced by a single-event transient (SET), not taking them into account in the SEU error rate. This is a reasonable assumption due to the low probability of two ions hitting the sensitive area in a polling period of 28.5 ms. These multiple errors were observed for LET values above $40 \mathrm{MeV} \cdot \mathrm{cm}^{2} / \mathrm{mg}$. All the SET errors, including multiple bit errors, were included as part of the corresponding ADC error rates.

Fig. 20 shows the experimental SEU cross-section, including a Weibull fit of the data points. The SEU LET threshold $\left(\mathrm{LET}_{\mathrm{th}}\right)$ is around $16.5 \mathrm{MeV} \cdot \mathrm{cm}^{2} / \mathrm{mg}$. Using an IRPP methodology [28], the estimated upset rate, for a $90 \%$ worst case geostationary orbit with 100 mils of Al shielding, is approximately $3.6 \cdot 10^{-8}$ errors/bit $\cdot$ day.

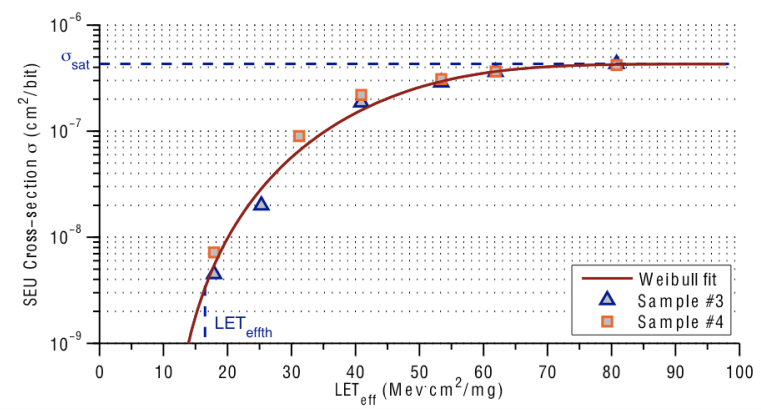

Fig. 20. Experimental SEU cross-section data with a Weibull fit. 


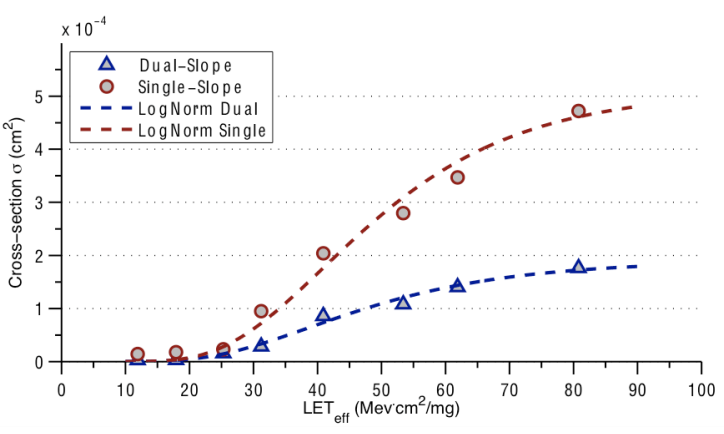

Fig. 21. Cross-section of DS ADC and SS ADC for errors larger than $4 \sigma$.

No single-event latchups (SELs) were produced, despite having used the maximum ion energy available $(80.8$ $\mathrm{MeV} \cdot \mathrm{cm}^{2} / \mathrm{mg}$ ). Fig. 21 shows the cross-sections obtained for the DS ADC and the SS ADCs with a log-normal approximation using least-square fit of the data points [29]. The saturation values obtained are $2.63 \cdot 10^{-4} \mathrm{~cm}^{2}$ for the dualslope, and $6.70 \cdot 10^{-4} \mathrm{~cm}^{2}$ for the single-slope topology. To obtain these data, it has been considered that a SEE error has been induced when the output data had a deviation larger than $4 \sigma$, where $\sigma$ corresponds with the ADC rms noise error. The larger cross-section for the SS ADC is partially explained by the effect of the ion-hits in the adaptive loop of the ramp generator circuit. In addition, and depending on the specific impact place of the particle, the fully-differential topology of the DS ADCs, may also contribute to minimize the effects of the ion-hit.

The estimated SEE error rate for the application example of a geostationary orbit with a maximum incident flux of cosmic rays, and assuming 100 mils of aluminum shielding, is of $2.1 \cdot 10^{-5} /$ day for the DS ADCs, and $3.4 \cdot 10^{-5} /$ day for each conversion channel of the SS ADC.

Temperature tests were performed by using the instrument Thermonics T-2650BV in the range between -25 and $125^{\circ} \mathrm{C}$. It was not possible to continue with the characterization for temperatures below $-25^{\circ} \mathrm{C}$ because the formation of ice crystals causing shorts in the ASIC. There were no significant deviations with respect to the performance results in Table I and Table II, apart of the expected increment in the thermal noise due to (7). The SS ADC, employed for the temperature monitoring, exhibited very high levels of stability against the extended temperature range. Fig. 22 shows the deviation of the output code against temperature with respect to the typical value at $25{ }^{\circ} \mathrm{C}$. The bandgap voltage was also measured and shown in the same conditions. As can be seen, the behavior of the stability against the temperature correlates again with the fluctuation of the bandgap voltage, with a maximum deviation less than $0.8 \%$. This means that the adaptation loop implemented in the SS ADC is working properly. This minor deviation can also be cancelled by performing ratiometric measurements. Similar behavior is expected for lower temperatures, and due to the previous characterization of the individual components in the selected CMOS technology [24]. This characterization was performed for temperatures down to $-110^{\circ} \mathrm{C}$ by using a thermal chamber. The experimental results showed that the coefficients of the standard design kit model

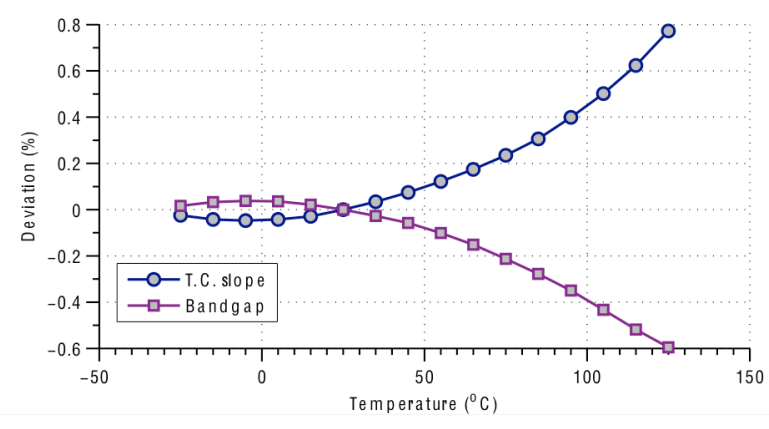

Fig. 22. Code stability of the SS ADC with $\mathrm{T}^{\mathrm{a}}$.

adequately the behavior of CMOS transistors over the extended range. The behavior of the FEE was also validated with simulations between -90 and $125{ }^{\circ} \mathrm{C}$ during the design process.

\section{CONCLUSIONS}

The proposed on-chip FEE, designed in a standard $0.35 \mu \mathrm{m}$ CMOS process, ensures reliable and stable operation under extreme environments, in terms of radiation and temperature, while maintaining the performance for high-resolution applications up to 16 bits. With the aim to provide a flexible solution, covering a higher range of sensors and applications, the FEE allows to configure the resolution, conversion rate, and the input voltage range, allowing at the same time to perform measurements with both deflection and comparison methods. In addition, the system includes precise thermal monitoring, sensor conditioning and error detection functionalities in order to increase the accuracy and the reliability. Experimental results, with a TID robustness beyond $300 \mathrm{krad}$ and a single-event error rate in the order of magnitude of $10^{-5}$ errors/day per conversion channel, allow to conclude that the proposed topologies and techniques to improve the linearity and the stability, in conjunction with the applied rad-hard techniques in the standard CMOS process, are reliable enough to be used in long-term space and nuclear applications without a significant loss in the performance.

\section{ACKNOWLEDGMENT}

This work has been supported by the Spanish Plan Nacional de Investigación of the Ministerio de Economía y Competitividad under projects MEIGA (AYA2009-14212-C05-04 and AYA2008-06420-C04-02), CISMAE (AYA2011-29967-C0505), and DANTE (TEC2011-28302), in turn partially funded by FEDER.

\section{REFERENCES}

[1] J. D. Cressler and H. A. Mantooth, Extreme Environment Electronics. Boca Raton, FL: CRC Press, 2013.

[2] L. Rockett, D. Patel, S. Danziger, B. Cronquist and J.J. Wang, "Radiation Hardened FPGA Technology for Space Applications," in Proc. IEEE Aerospace Conf., 2007. doi: 10.1109/AERO.2007.353098

[3] G. Furano, R. Jansen and A. Menicucci, "Review of Radiation Hard Electronics Activities at European Space Agency," Journal of Instrumentation, vol. 8, no. 02, p. C02007, Feb. 2013.

[4] P. C. Adell, J. Yager, Z. Pannell, J. Shelton, M. M. Mojarradi, B. Blalock, et al., "Radiation Hardening of an SiGe BiCMOS Wilkinson 
ADC for Distributed Motor Controller Application," IEEE Trans. Nucl. Sci., vol. 61, no. 3, pp. 1236-1242, Jun. 2014.

[5] C. Ulaganathan, N. Nambiar, K. Cornett, R. L. Greenwell, J. A. Yager, B. S. Prothro, et al., "A SiGe BiCMOS Instrumentation Channel for Extreme Environment Applications," VLSI Design, vol. 2010, no. 3, Jan. 2010, Art. ID 156829.

[6] R. M. Diestelhorst, T. D. England, R. Berger, R. Garbos, C. Ulaganathan, B. Blalock, et al., "A new approach to designing electronic systems for operation in extreme environments: Part I - The SiGe Remote Sensor Interface," IEEE Trans. Aerosp. Electron. Syst. Mag., vol. 27, no. 6, pp. 25-34, Jun. 2012.

[7] R. C. Lacoe, "Improving Integrated Circuit Performance Through the Application of Hardness-by-Design Methodology," IEEE Trans. Nucl. Sci., vol. 55, no. 4, pp. 1903-1925, Aug. 2008.

[8] W. Snoeys, F. Faccio, M. Burns, M. Campbell, E. Cantatore, N. Carrer, et al., "Layout techniques to enhance the radiation tolerance of standard CMOS technologies demonstrated on a pixel detector readout chip," Nuclear Instruments and Methods in Physics Research, vol. 439, no. 23, pp. 349-360, Jan. 2000.

[9] T. R. Oldham and F. B. McLean, "Total Ionizing Dose Effects in MOS Oxides and Devices," IEEE Trans. Nucl. Sci., vol. 50, no. 3, pp. 483499, Jun. 2003.

[10] H. J. Barnaby, "Total-ionizing-dose effects in modern CMOS technologies," IEEE Trans. Nucl. Sci., vol. 53, no. 6, pp. 3103-3121, Dec. 2006.

[11] H. L. Hughes and J. M. Benedetto, "Radiation effects and hardening of MOS technology: devices and circuits," IEEE Trans. Nucl. Sci., vol. 50, no. 3, pp. 500-521, Jun. 2003.

[12] C. C. Chen, S. C. Liu, C. C. Hsiao and J. G. Hwu, "A circuit design for the improvement of radiation hardness in CMOS digital circuits," IEEE Trans. Nucl. Sci., vol. 39, no. 2, pp. 272-277, Apr. 1992.

[13] C. Feng, D. Zhang, J. Zhang, S. Gao, D. Yang, Y. Zhang, et al., "Design of the Readout Electronics for the BGO Calorimeter of DAMPE Mission," IEEE Trans. Nucl. Sci., vol. 62, no. 6, pp. 3117-3125, Dec. 2015.

[14] H. Nakajima, D. Matsuura, T. Idehara, N. Anabuki, H. Tsunemi, J. Doty, et al., "Development of the analog ASIC for multi-channel readout Xray CCD camera," Nuclear Instruments and Methods in Physics Research Section A: Accelerators, Spectrometers, Detectors and Associated Equipment, vol. 632, no. 1, pp. 128-132, Feb. 2011.

[15] K. V. Tham, C. Ulaganathan, N. Nambiar, R. L. Greenwell, C. L. Britton, M. N. Ericson, et al., "PVT compensation for Wilkinson singleslope measurement systems," IEEE Trans. Nucl. Sci., vol. 59, no. 5, pp. 2444-2450, Oct. 2012.

[16] G. Shan-Shan, F. Chang-Qing, D. Jiang, L. Shu-Bin, Z. G. ZHANG, X. I. Kai and A. N. Qi, "Radiation tolerance studies on the VA32 ASIC for DAMPE BGO calorimeter," Nuclear Science and Techniques, vol. 25, no. 5, pp. 010402(1)-010402(5), 2014.

[17] T. Vergine, M. De Matteis, L. Rota, A. Marchioro, and A. Baschirotto, "An automatic calibration circuit for 12-bits single-ramp A-to-D converter in LHC environments," in Proc. 9th Conference on Ph.D. Research in Microelectronics and Electronics (PRIME), 2013, pp. 4548.

[18] I. Arruego, "A Roadmap for the Development of Miniature Instrumentation for Mars Exploration," in Proc. Int. Workshop on Instrum. for Planetary Missions, Greenbelt, Maryland, 2014. [Online]. Available: http://ssed.gsfc.nasa.gov/IPM/PDF/1068.pdf. [Accessed Jan. $25,2016]$.

[19] J. A. Rodríguez-Manfredi, M. de la Torre, N. T. Bridges, P. G. Conrad, F. Ferri, M. Genzer, et al., "MEDA, an Environmental and Meteorological Package for the Mars 2020 Mission," in Proc. Int. Workshop on Instrum. for Planetary Missions, Greenbelt, Maryland, 2014. [Online]. Available: http://ssed.gsfc.nasa.gov/IPM/PDF/1125.pdf. [Accessed Jan. 25, 2016].

[20] V. Agarwal and S. D. Birkar, "Comparison of gamma radiation performance of a range of CMOS A/D converters under biased conditions," IEEE Trans. Nucl. Sci., vol. 52, no. 6, pp. 3059-3067, Dec. 2005.

[21] S. Sordo-Ibáñez, B. Piñero-García, M. Muñoz-Díaz, A. Ragel-Morales, J. Ceballos-Cáceres, L. Carranza-González, et al., "A rad-hard multichannel front-end readout ASIC for space applications," in Proc. IEEE Workshop on Metrology for Aerospace, Benevento, 2014, pp. 180184.

[22] S. Sordo-Ibáñez, S. Espejo-Meana, B. Piñero-García, A. Ragel-Morales, J. Ceballos-Cáceres, M. Muñoz-Díaz, et al., "Four-channel self- compensating single-slope ADC for space environments," Electron. Lett., vol. 50, no. 8, pp. 579-581, Apr. 2014.

[23] S. Sordo-Ibáñez, B. Piñero-García, M. Muñoz-Díaz, A. Ragel-Morales, J. Ceballos-Cáceres, L. Carranza-González, et al., "A Front-End ASIC for a 3-D Magnetometer for Space Applications by Using Anisotropic Magnetoresistors," IEEE Trans. Magn., vol. 51, no. 1, Jan. 2015, Art. ID 4001804.

[24] J. Ramos-Martos, A. Arias-Drake, A. Ragel-Morales, J. CeballosCáceres, J. M. Mora-Gutiérrez, B. Piñero-García, et al., "Radiation characterization of the austriamicrosystems $0.35 \mu \mathrm{m}$ CMOS technology," in Proc. 12th Eur. Conf. on Radiation and Its Effects on Components and Systems, Seville, Spain, 2011, pp. 806-811.

[25] H. J. Barnaby, "Total dose effects in linear bipolar integrated circuits," International journal of high speed electronics and systems, vol. 14, no. 02, pp. 519-541, 2004.

[26] K. Kruckmeyer, J. S. Prater, B. Brown, and T. Trinh, "Analysis of low dose rate effects on parasitic bipolar structures in CMOS processes for mixed-signal integrated circuits," IEEE Trans. Nucl. Sci., vol. 58, no. 3, pp. 1023-1031, Jun. 2011.

[27] H. Barnaby, H. J. Tausch, R. Turfler, P. Cole, P. Baker, and R. L. Pease, "Analysis of bipolar linear circuit response mechanisms for high and low dose rate total dose irradiations," IEEE Trans. Nucl. Sci., vol. 43, no. 6, pp. 3040-3048, Dec. 1996.

[28] E. L. Petersen, "The SEU figure of merit and proton upset rate calculations," IEEE Trans. Nucl. Sci., vol. 45, no. 6, pp. 2550-2562, Dec. 1998.

[29] J. Ramos-Martos, A. Arias-Drake, L. Carranza-González, S. SordoIbáñez, J. Ceballos-Cáceres, J. M. Mora-Gutiérrez, et al., "SEE characterization of a magnetometer front-end ASIC using a RHBD digital library in AMS $0.35 \mu \mathrm{m}$ CMOS," in Proc. 5th Int. Workshop on Analogue and Mixed-Signal Integrated Circuits for Space Applications, Geneva, Switzerland, 2014. 